\title{
miR-122 Targets X-Linked Inhibitor of Apoptosis Protein to Sensitize Oxaliplatin- Resistant Colorectal Cancer Cells to Oxaliplatin-Mediated Cytotoxicity
}

\author{
Yongqiang Hua a,b Yaodong Zhu ${ }^{\mathrm{c}}$ Jijie Zhang ${ }^{\mathrm{d}}$ Zhenfeng Zhu ${ }^{\mathrm{a}, \mathrm{b}}$ \\ Zhouyu Ning ${ }^{a, b}$ Hao Chen ${ }^{a, b}$ Luming Liu ${ }^{a, b}$ Zhen Chen ${ }^{a, b}$ \\ Zhiqiang Menga,b \\ aMinimally Invasive Treatment Center, Fudan University Shanghai Cancer Center, Shanghai, \\ ${ }^{b}$ Department of Oncology, Shanghai Medical College, Fudan University, Shanghai, 'Chinese Integrative \\ Medicine Oncology Department, First Affiliated Hospital of Medical University of Anhui, Hefei City, \\ 'Oncology Department, Danyang People's Hospital of Jiangsu Province, Danyang City, Jiangsu, China
}

\section{Key Words}

Colorectal cancer $\bullet$ Oxaliplatin resistance $\cdot \operatorname{miR}-122 \cdot$ XIAP

\begin{abstract}
Background/Aims: Although oxaliplatin is one of the most effective chemotherapeutic drugs used to treat colorectal cancer (CRC), long-term administration usually induces acquired drug resistance during the course of treatment. Thus, there is an urgent need to explore novel strategies to improve the efficiency of cancer therapy. The aim of this study was to explore the effect of microRNA-122 (miR-122) on reversing oxaliplatin resistance in CRC. Methods: The expression of miR-122 in CRC cells was examined by quantitative reverse transcriptase real-time PCR. The cytotoxicity of oxaliplatin against CRC cells was evaluated by Cell Counting Kit- 8 assays. Mitochondrial membrane potentials and cell apoptotic rates were measured by flow cytometry. Cellular protein expression and interactions were detected by western blot and co-immunoprecipitation. Results: Established oxaliplatin-resistant SW480 and HT29 cells (SW480/OR and HT29/OR) expressed significantly higher levels of X-linked inhibitor of apoptosis protein (XIAP) and lower levels of miR-122 compared with normal SW480 and HT29 cells, respectively. Our results showed that the downregulation of miR-122 was responsible for the overexpression of XIAP in these oxaliplatin-resistant CRC cells. We then found that the recovery of miR-122 expression can sensitize SW480/OR and HT29/OR cells to oxaliplatinmediated apoptosis through the inhibition of XIAP expression. Conclusion: Upregulation of $\mathrm{XIAP}$ in CRC cells is responsible for the acquired resistance to oxaliplatin. Furthermore, miR122 reversed oxaliplatin resistance in CRC by targeting XIAP.




\section{Introduction}

Colorectal cancer (CRC) is the third most common malignancy worldwide. Despite the advances made in the diagnosis and treatment strategies used for CRC in recent decades, the prognosis for CRC remains poor because it is aggressive and has a high metastatic potential $[1,2]$. Platinum-based chemotherapeutic drugs are still commonly used for the treatment of CRC; however, CRC cells usually acquire drug resistance because of the longterm and repeated use of chemotherapeutic drugs [3-5]. We aimed to explore the potential mechanisms and strategies that could improve the efficiency of cancer therapies.

Recent studies have indicated that the dysregulation of microRNAs (miRNAs) often occurs and is responsible for the development of drug resistance in cancers. miRNAs are non-coding RNAs, about 19-25 nucleotides long. miRNAs regulate the expression of a wide variety of genes by binding to the $3^{\prime}$ untranslated region ( $3^{\prime}$ UTR) of targeted mRNAs, leading to mRNA degradation or the inhibition of protein translation [6-8]. Since approximately $60 \%$ of human genes are regulated by miRNAs, these molecules are reported to play important roles in tumorigenesis, tumor growth and survival, and drug resistance. The correction of miRNA disorders may represent a potential strategy for reversing chemoresistance [9-11].

Oxaliplatin is a third-generation platinum-based chemotherapeutic drug that is commonly used for the treatment of CRC. Oxaliplatin induces apoptosis in CRC cells by damaging cellular DNA through the formation of intrastrand guanine-guanine and guanineadenine DNA links $[12,13]$. However, under the long-term use of oxaliplatin, cancer cells usually change their gene expression profile to acquire drug resistance against oxaliplatininduced apoptosis [14-16]. Chemoresistance has become a major obstacle in the clinical use of oxaliplatin among CRC patients; thus, we aim to attenuate the acquired resistance to improve the curative effect of oxaliplatin.

\section{Materials and Methods}

\section{Cell culture}

Human CRC cell lines SW480 and HT29 were obtained from the American Type Culture Collection (Rockville, MD) and maintained in RPMI-1640 medium supplemented with 10\% fetal bovine serum (Gibco, Invitrogen, Carlsbad, $\mathrm{CA}$ ) in an incubator with $5 \% \mathrm{CO}_{2}$ at $37{ }^{\circ} \mathrm{C}$. To establish oxaliplatin-resistant CRC models, SW480 and HT29 cells were exposed to increasing concentrations of oxaliplatin (Sigma-Aldrich, Darmstadt, Germany). Briefly, SW480 and HT29 cells were initially treated with oxaliplatin at $0.1 \mu \mathrm{M}$ for 1 month. Subsequently, the oxaliplatin concentration in the culture medium was increased every 2 weeks by $0.1 \mu \mathrm{M}$ up to a final concentration of $1 \mu \mathrm{M}$. The established oxaliplatin-resistant SW480 and HT29 cells were named SW480/OR and HT29/OR, respectively. To eliminate the influence of residual oxaliplatin in the culture medium, SW480/OR and HT29/OR cells were cultured in oxaliplatin-free RPMI-1640 for 2 weeks prior to the experiments.

\section{Quantitative reverse transcriptase real time PCR ( $q R T-P C R)$}

Relative expression of miR-122 was tested via qRT-PCR analysis. Briefly, we extracted the total RNAs of CRC cell lines and tissues by using TRIzol® reagent (Thermo Fisher Scientific, Inc., Waltham, MA). Subsequently, reverse transcription of total RNA was performed via a stem-loop RT primer (5'-CTCAACTGGTGTCGTGGAGTCGGCAATTCAGTTGAGACA AACAC-3', Ribobio, Guangzhou, China) and the PrimeScript RT reagent kit, according to the manufacturer's instructions (Takara Bio, Inc., Otsu, Japan). The amplification of miR-122 was then performed via the SYBR Premix Ex Taq (Takara Bio) on an Applied Biosystems 7500 Sequence Detection system (Applied Biosystems, Foster City, CA). 


\section{Cell transfection}

Recombinant pcDNA3.1 plasmid carrying X-linked inhibitor of apoptosis protein (XIAP) open reading frame $(2 \mu \mathrm{g} / \mathrm{mL})$, hsa-miR-122 mimic (miR-122, GenePharma Co., Ltd., Shanghai, China) (5'-UGGAGUGUACAAUGGUGUUUGU-3') (50 pmol/mL), miR-122 inhibitor (anti-miR-122, GenePharma Co., Ltd.) (5'-ACAAACACCAUUGUACACUCCA-3') (50 pmol/mL), negative control oligonucleotide (GenePharma Co., Ltd.) (5'-GGGUUAAUGUGCAUGUUUGAGU-3') (50 pmol/mL) and XIAP small interfering RNA (XIAP siRNA; GenePharma Co., Ltd.) (50 pmol/mL) were transfected into cells via Lipofectamine 2000 (Invitrogen) according to the manufacturer's instructions.

\section{Luciferase reporter assay}

XIAP 3' UTR fragment containing predicted miR-122 binding site was amplified and inserted into the downstream of firefly luciferase gene in the luciferase reporter pGL3 Luciferase Reporter Vectors (Promega, Madison, WI). The recombinant plasmid was named pGL3-wild XIAP. The mutant XIAP reporter was created by mutating the seed regions of the miR-122 binding sites (CACUCCA) via the site-directed mutagenesis kit (Takara Bio) and named pGL3-mutant XIAP. To perform the luciferase reporter assay, luciferase reporter vectors $(2 \mu \mathrm{g} / \mathrm{mL})$, Renilla luciferase pRL-TK plasmid (Promega) (100 ng/mL), miR-122 (50 pmol/mL), and anti-miR-122 (50 pmol/mL) were co-transfected into cells by using Lipofectamine 2000. Luciferase activities were measured after 48 of incubation with the Dual-Luciferase Reporter assay system (Promega) according to the manufacturer's instructions.

\section{Cytotoxicity assays}

CRC cells were treated with different concentrations of oxaliplatin with or without transfection. Following $48 \mathrm{~h}$ of incubation, the cytotoxicity of oxaliplatin in CRC cells was evaluated by Cell Counting Kit-8 (CCK-8) assays. Briefly, $10 \mu \mathrm{L}$ of CCK-8 solution was added to the cells followed by incubation at $37^{\circ} \mathrm{C}$ for 2 h. Absorbance at $450 \mathrm{~nm}$ was measured via an enzyme-linked immunosorbent assay microplate reader. The half maximal inhibitory concentration $\left(\mathrm{IC}_{50}\right.$ ) of oxaliplatin against $\mathrm{CRC}$ cells was calculated according to the cell viability of SW480 and OR-SW480 cells.

\section{Co-immunoprecipitation}

Cells were collected and incubated with lysis buffer (Cell Signaling Technologies, Danvers, MA) on ice. Supernatants were subsequently collected by centrifugation for $5 \mathrm{~min}$ at $12,000 \times \mathrm{g}$. After incubation with the primary antibody XIAP (Cell Signaling Technologies) overnight at $4{ }^{\circ} \mathrm{C}$, protein $\mathrm{G}$ agarose beads were added for $2 \mathrm{~h}$. Immunoprecipitates were then washed with cold lysis buffer and boiled with sodium dodecyl sulfate sample buffer.

\section{Western blot analysis}

CRC cells were collected and washed. Total proteins were then extracted with RIPA lysis buffer. Equal amount of extracted proteins or immunoprecipitates were separated by $10 \%$ sodium dodecyl sulfate-polyacrylamide gel electrophoresis followed by transfer to polyvinylidene fluoride membranes. Subsequently, the membranes were blocked with 5\% non-fat milk at room temperature for $1 \mathrm{~h}$ and then incubated with antibodies (Cell Signaling Technology) against XIAP, caspase-9, caspase-3, and GAPDH overnight at $4{ }^{\circ} \mathrm{C}$. After incubation with horseradish peroxidase-conjugated second antibody (Cell Signaling Technology), membranes were washed and probed by the enhanced chemiluminescence system (Thermo Fisher Scientific).

\section{Measurement of cell apoptosis}

After the treatments, CRC cells were collected and washed. Cell apoptosis was measured by the Annexin V-FITC apoptosis detection kit (Sigma-Aldrich) via flow cytometry, according to the manufacturer's instruction. 
Tumor growth in nude mice

SW480/OR cells stably expressing the miR-122 sequence (LT-miR-122) were developed via a lentiviralbased system (Genechem Co., Ltd., Shanghai, China) for tumorigenesis assays. Immunodeficient nude BALB/c mice (4-6 weeks old, $n=28$ ) were purchased from Shanghai Super-B\&K Laboratory Animal Corp., Ltd. (Shanghai, China). Xenograft tumors were generated via subcutaneous injection of SW480/OR cells. After the xenografts reached $0.5 \mathrm{~cm}$ in diameter, the animals were treated with intraperitoneal oxaliplatin twice a week $(5 \mathrm{mg} / \mathrm{kg})$.The mice were sacrificed after 28 days and the tumor tissues were harvested. The animal care and experimental protocols were approved by the Animal Care Committee of Shanghai Medical College, Fudan University.

\section{Statistical analysis}

Data are expressed as the mean \pm standard deviation obtained from three independent experiments. For comparison analyses, two-tailed Student's t-tests were used to estimate the statistical differences between two groups. One-way analysis of variance was used to determine the differences between three or more groups. Statistical analysis was performed using SPSS 16.0 software (SPSS Inc., Chicago, IL). We considered $p<0.05$ to indicate a statistically significant difference.

\section{Results}

\section{SW480/OR and HT29/OR cells are resistant to oxaliplatin treatment}

To study the oxaliplatin resistance of CRC cells, we established oxaliplatin-resistant CRC models in SW480 and HT29 (SW480/OR and HT29/OR) cells through extended exposure to oxaliplatin. We observed that SW480/OR cells exhibited obvious oxaliplatin resistance compared with their parental SW480 cells. Furthermore, the $\mathrm{IC}_{50}$ of oxaliplatin to SW480/ OR increased by 10.2 -fold compared with the SW480 cells (Fig. 1A). Similarly, the $\mathrm{IC}_{50}$ of oxaliplatin against HT29/ OR cells increased by 9.2 -fold compared with the parental HT29 cells (Fig. 1B). These results indicated that our established SW480/OR and HT29/OR cells were resistant to oxaliplatin treatment.

\section{Upregulation of XIAP is responsible for oxaliplatin resistance in SW480/OR and HT29/OR \\ Previous studies} have indicated that the overexpression of XIAP contributes to the development of drug-resistance in multiple cancers [17-19]. We therefore investigated the potential role of XIAP in SW480/OR and HT29/OR cells. Interestingly,

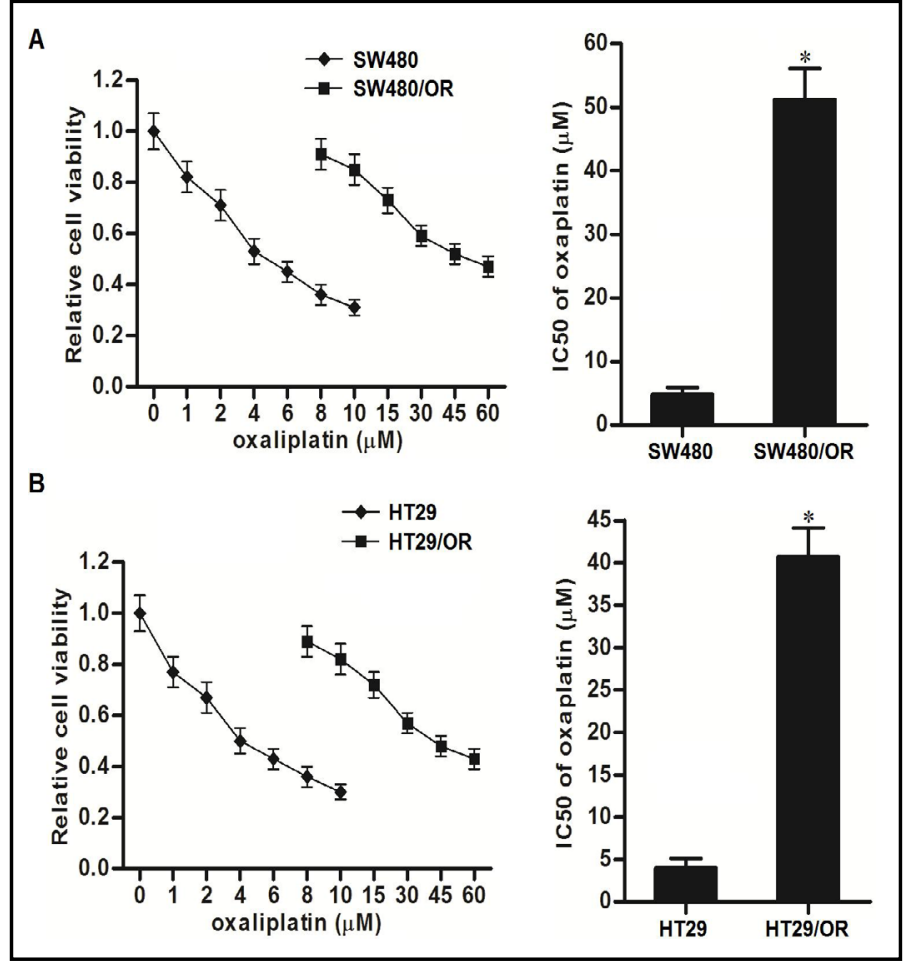

Fig. 1. Resistance of SW480/OR and HT29/OR against oxaliplatin. A. SW480 and SW480/OR cells were treated with different concentrations of oxaliplatin before CCK-8 assays. ${ }^{*} \mathrm{p}<0.05$ vs. SW480 cells. B. HT29 and HT29/OR cells were treated with different concentrations of oxaliplatin before CCK- 8 assays. ${ }^{*}$ p $<0.05$ vs. HT2 9 cells. 
Fig. 2. Role of XIAP in the changes of sensitivity among CRC cells to oxaliplatin treatment. A. Expression of XIAP in SW480, SW480/OR, HT29, and HT29/OR cells was evaluated by western blot analysis. B. Effect of XIAP siRNA on changes in the protein levels of XIAP in SW480/OR and HT29/ OR. C. Effect of XIAP plasmid on changes in the protein levels of XIAP in SW480 and HT29. D. Effect of XIAP siRNA on changes in the sensitivity of SW480/OR and HT29/OR to oxaliplatin $(10 \mu \mathrm{M})$ treatment. ${ }^{*} \mathrm{p}<0.05$ vs. the oxaliplatin + negative control oligonucleotide (NCO) group. E. Effect of XIAP plasmid on changes in the sensitivity of SW480 and HT29 to oxaliplatin $(10 \mu \mathrm{M})$ treatment. $* \mathrm{p}<0.05$ vs. the oxaliplatin + NCO group.



we observed that SW480/OR and HT29/OR cells expressed higher levels of XIAP compared to their parental SW480 and HT29 cells, respectively (Fig. 2A). We then performed a loss-offunction assay of XIAP in SW480/OR and HT29/OR cells by transfection with XIAP siRNA, and a gain-of-function assay of XIAP in SW480 and HT29 cells by using XIAP plasmid. The transfection efficiencies of XIAP siRNA and XIAP plasmids are shown in Fig. 2B and 2C. Interestingly, our results showed that knockdown of XIAP in SW480/OR and HT29/OR cells significantly increased cell sensitivity to oxaliplatin treatment (Fig. 2D). On the other hand, SW480 and HT29 cells obtained resistance to oxaliplatin after transfection with XIAP plasmid (Fig. 2E). Taken together, we have demonstrated that the overexpression of XIAP contributed to the development of oxaliplatin resistance in SW480/OR and HT29/OR cells. Furthermore, XIAP can be considered a potential target for reducing oxaliplatin resistance in CRC. 


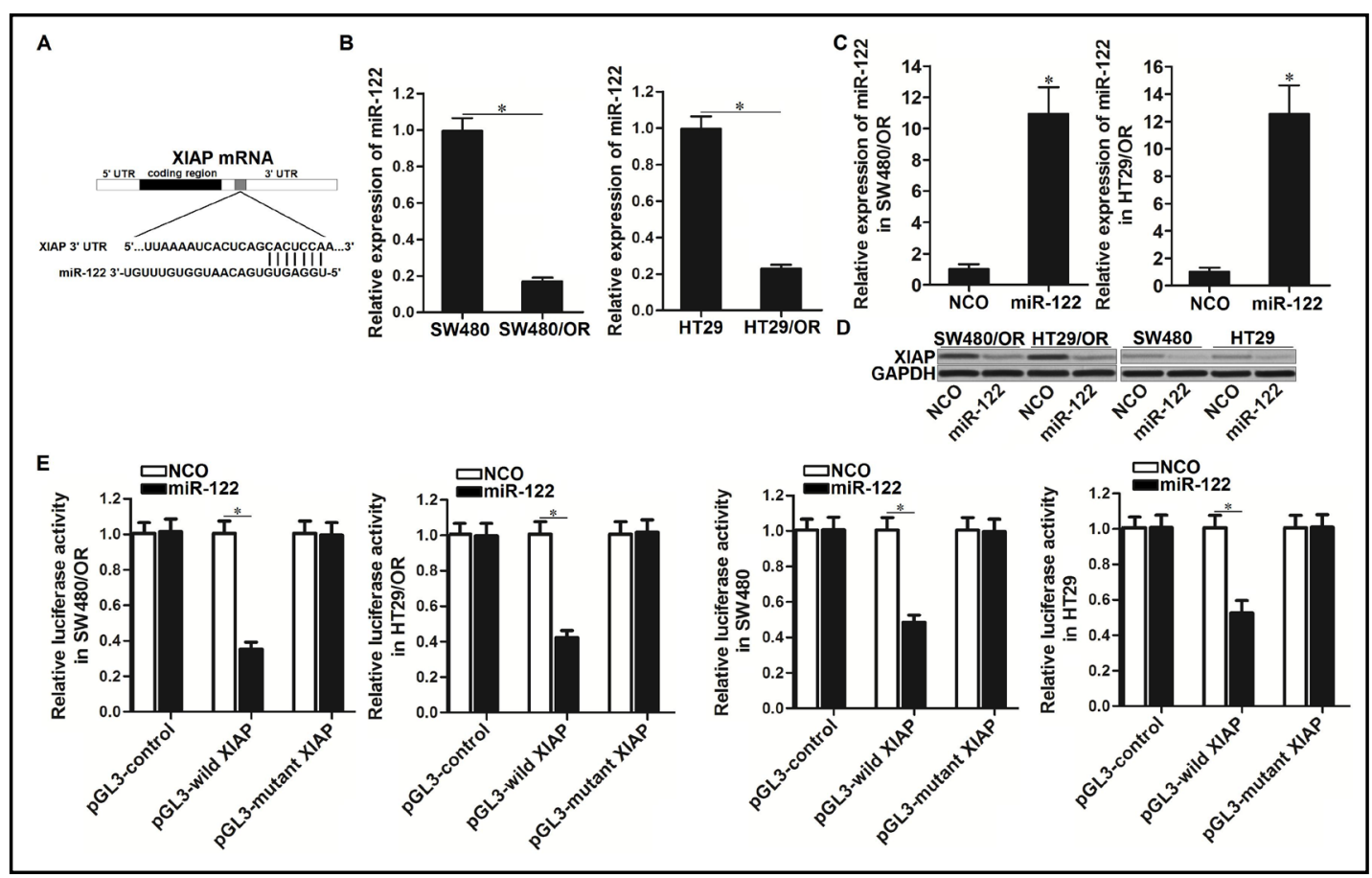

Fig. 3. XIAP is the target of miR-122. A. Seed region of XIAP $3^{\prime}$ UTR paired with miR-122. B. Relative expression of miR-122 in SW480, SW480/OR, HT29, and HT29/OR cells. ${ }^{*} \mathrm{p}<0.05$. C. Transfection efficiency of miR-122 mimics in SW480/OR and HT29/OR cells. ${ }^{*} \mathrm{p}<0.05$ vs. the NCO group. D. Effect of miR-122 mimics on changes in the protein levels of XIAP in SW480/OR, HT29/OR, SW480, and HT29 cells. E. After co-transfection with miR-122 and pGL3 luciferase reporters, luciferase activities were measured via the Dual-Luciferase Reporter Assay System according to the manufacturer's instructions. ${ }^{*} \mathrm{p}<0.05$.

Overexpression of XIAP is induced by the absence of miR-122 in SW480/OR and HT29/OR

Previous studies have demonstrated that a change in gene expression profile is usually induced by the dysregulation of miRNAs in cancer cells. We therefore searched for related miRNAs that may be upstream of XIAP. The results of bioinformatics databases (TargetScan, miRanda, and PicTar) showed that the 3' UTR of XIAP mRNA contains a seed region paired with miR-122 (Fig. 3A). Furthermore, we found significant decreases of miR-122 expression in SW480/OR and HT29/OR cells compared with the parental SW480 and HT29 cells, respectively (Fig. 3B). We therefore speculated that the overexpression of XIAP may be induced by the absence of miR-122 in SW480/OR and HT29/OR cells. To investigate the miR-122/XIAP axis, we transfected SW480/OR and HT29/OR cells with miR-122 mimics (Fig. 3C). We then found that the recovery of miR-122 expression suppressed the expression of XIAP in SW480/OR, HT29/OR, SW480 and HT29 cells (Fig. 3D). Furthermore, the results of luciferase reporter assays showed that co-transfection with miR-122 decreased the luciferase activity of the pGL3 luciferase reporter carrying XIAP $3^{\prime}$ UTR. In contrast, miR-122 exhibited no effects on the empty pGL3 luciferase reporter or that carrying mutant XIAP $3^{\prime}$ UTR (Fig. 3E). Taken together, we demonstrated that the overexpression of XIAP was induced by the absence of miR-122 in SW480/OR and HT29/OR cells.

Recovery of miR-122 re-sensitizes SW480/OR and HT29/OR cells to oxaliplatin through the suppression of XIAP

To investigate the role of miR-122 in regulating oxaliplatin resistance in CRC, we transfected SW480/OR and HT29/OR cells with miR-122 mimics before treatment with oxaliplatin. The results of CCK- 8 assays showed that transfection with miR-122 significantly increased the sensitivity of SW480/OR cells to oxaliplatin treatment. Intuitively, IC $_{50}$ of oxaliplatin against SW480/OR was reduced by $78.9 \%$ because of the miR-122 treatment 


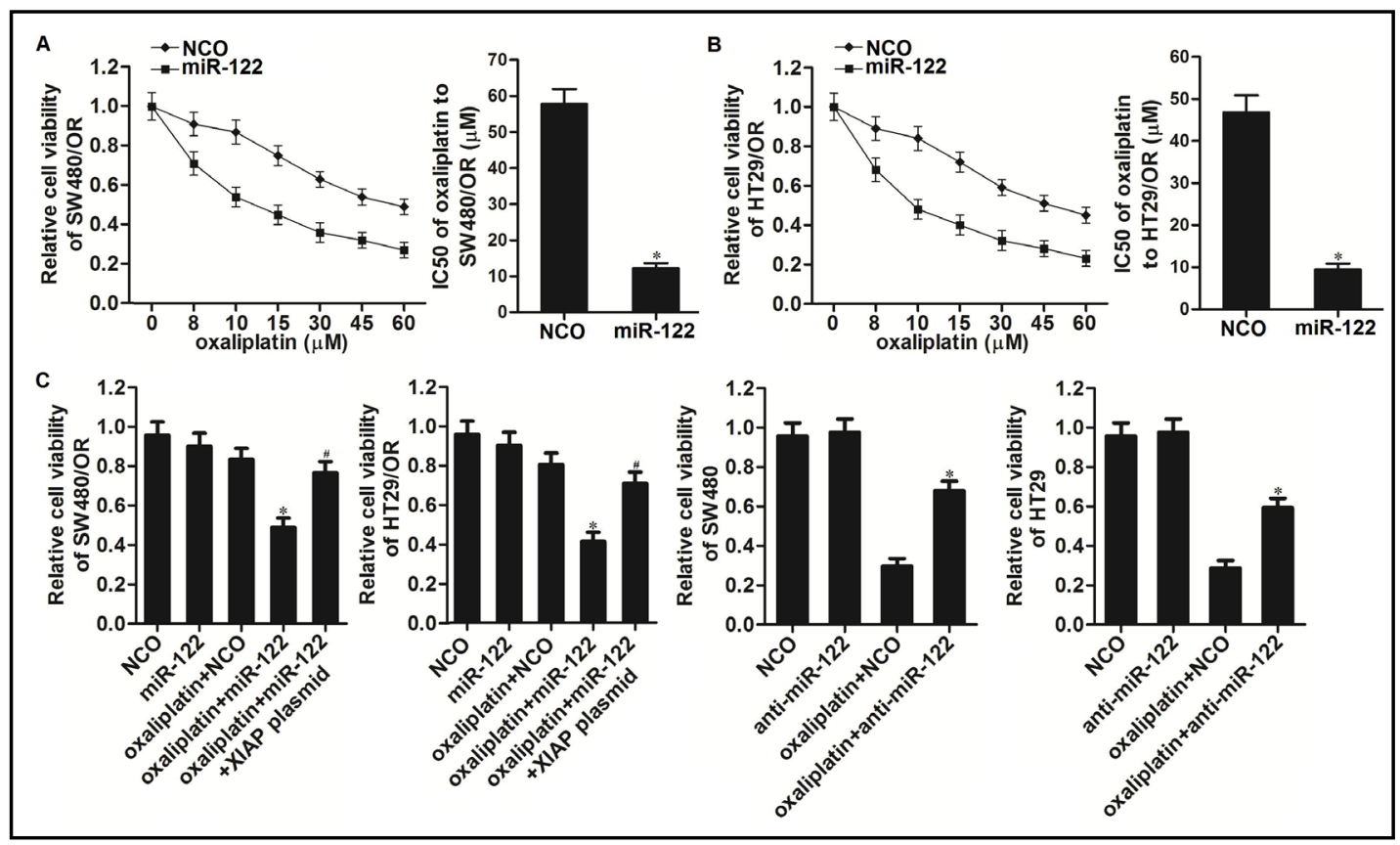

Fig. 4. miR-122 increased the cytotoxicity of oxaliplatin to SW480/OR and HT29/OR cells by targeting XIAP. A. NCO or miR-122-transfected SW480/OR cells were treated with oxaliplatin $(0-60 \mu \mathrm{M})$. Cell viability was measured by CCK- 8 assays. ${ }^{*}$ p $<0.05$ vs. the NCO group. B. NCO or miR-122-transfected HT29/OR cells were treated with oxaliplatin $(0-60 \mu \mathrm{M})$. Cell viability was measured by CCK- 8 assays. ${ }^{*} \mathrm{p}<0.05$ vs. the NCO group. C. NCO, anti-miR-122, miR-122 and XIAP plasmid-treated SW480/OR, HT29/OR, SW480, and HT29 cells were treated with oxaliplatin $(10 \mu \mathrm{M})$. Cell viability was measured by CCK-8 assays. ${ }^{*} \mathrm{p}<0.05$ vs. the oxaliplatin + NCO group. ${ }^{\#} \mathrm{p}<0.05$ vs. oxaliplatin + miR-122 group.

(Fig. 4A). Similarly, miR-122 decreased the $\mathrm{IC}_{50}$ of oxaliplatin to HT29/0R by $80.5 \%$ (Fig. 4B). However, under co-treatment with miR-122 and oxaliplatin, the cell viability of XIAP plasmid-transfected SW480/OR and HT29/OR cells was significantly higher than that of the empty plasmid-transfected SW480/OR and HT29/OR cells, respectively. Furthermore, we found that anti-miR-122 significantly decreased the sensitivity of normal SW480 and HT29 cells to oxaliplatin (Fig. 4C). These results suggested that miR-122 increased the cytotoxicity of oxaliplatin to SW480/OR and HT29/OR cells by targeting XIAP.

miR-122/XIAP axis regulates the sensitivity of SW480/OR and HT29/OR cells to oxaliplatininduce apoptosis

XIAP is a known suppressor of caspases [20,21]; thus we investigated the effect of miR-122 on oxaliplatin-induced apoptotic pathways in SW480/OR and HT29/OR cells. The results of co-immunoprecipitation and western blot analysis showed that co-treatment with miR-122 inhibited an interaction with XIAP and caspase-9, $(-3)$. However, the enforced expression of XIAP plasmid increased conjugation with XIAP and caspase-9, (-3) in SW480/ OR and HT29/OR cells co-treated with oxaliplatin and miR-122 (Fig. 5A). The results showed that co-treatment with miR-122 promoted the activation of caspase- 9 and caspase- 3 (Fig. 5B), which was followed by apoptosis (Fig. 5C) in the oxaliplatin-treated SW480/OR and HT29/OR cells.

miR-122 enhances the anti-tumor effect of oxaliplatin against CRC

To investigate the anti-tumor effect of miR-122 on oxaliplatin resistance in CRC in vivo, we established CRC mice models by inoculating SW480/OR cells, which stably expressed miR-122 sequence (LT-miR-122) or did not (LT-control). We found that the LT-control was not sensitive to oxaliplatin treatment. In contrast, oxaliplatin significantly reduced the size of 
A
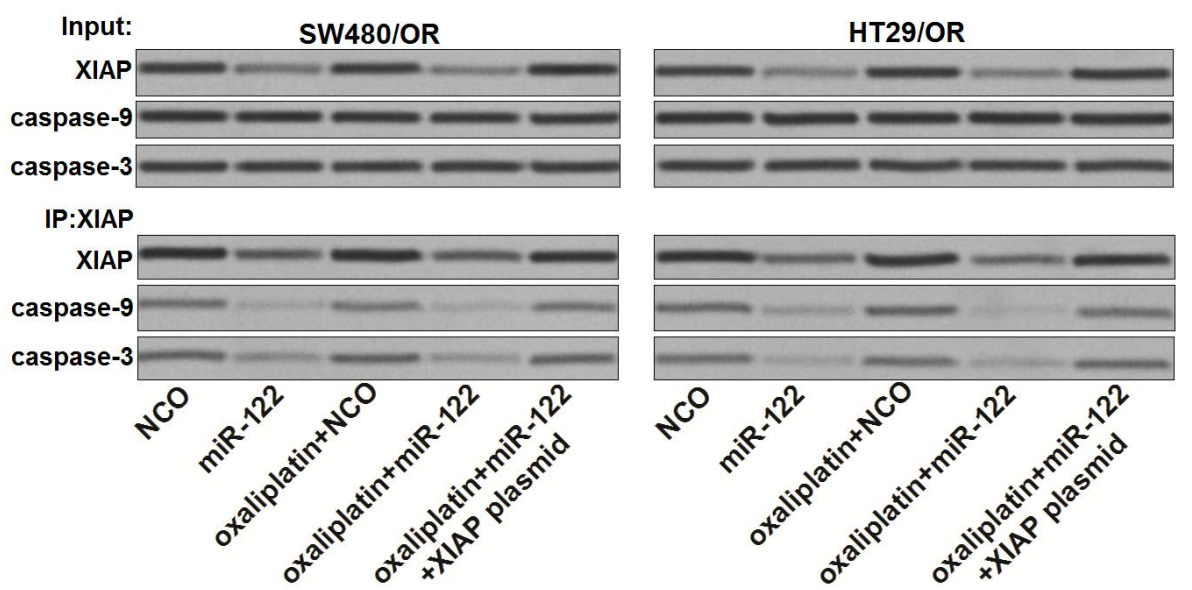

B
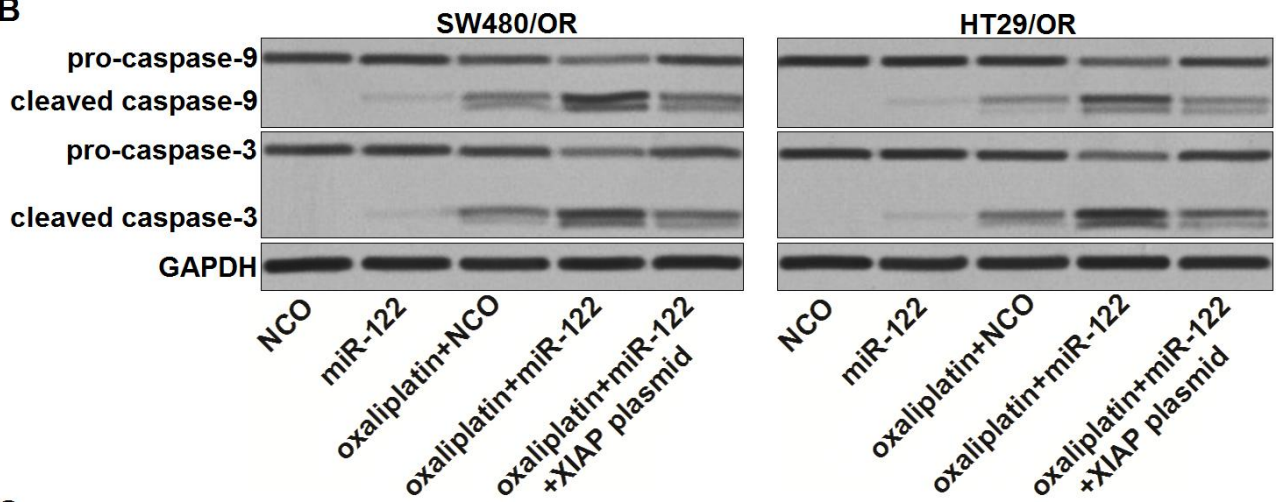

C
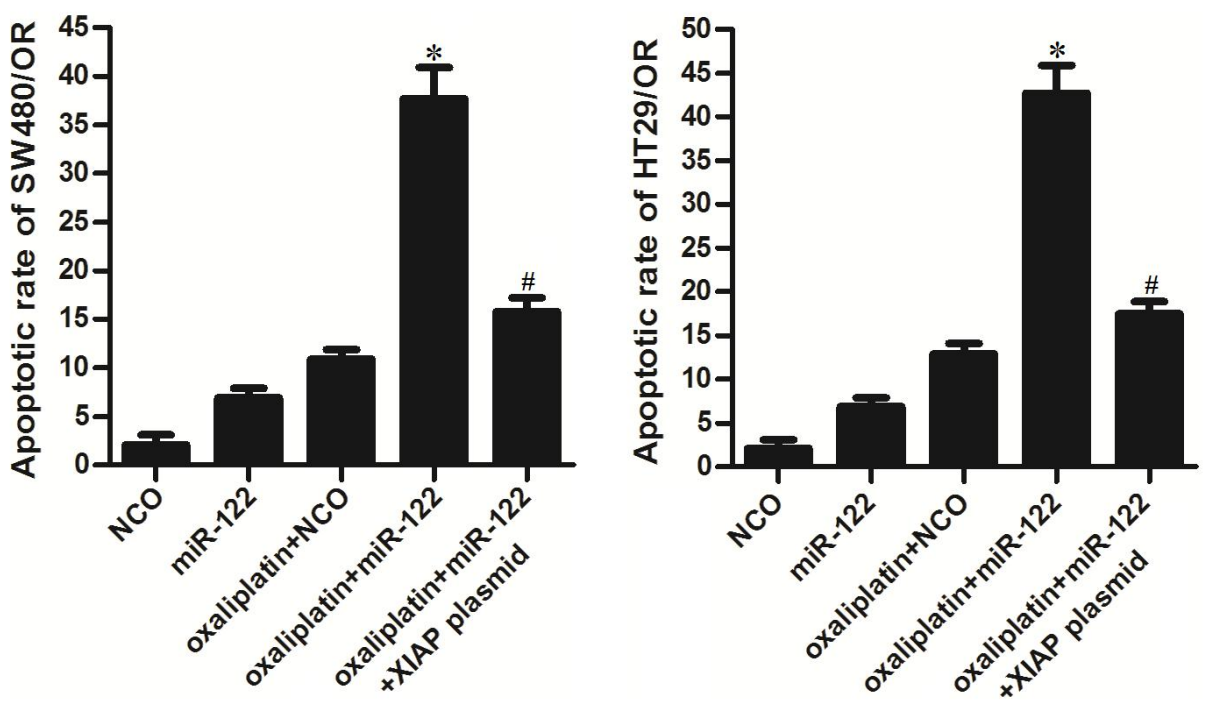

Fig. 5. miR-122 promoted oxaliplatin-induced apoptosis in SW480/OR and HT29/OR cells. A. Interaction of XIAP with caspase- 9 and caspase- 3 was detected by co-immunoprecipitation and western blot analysis in the SW480/OR and HT29/OR cells treated with miR-122 and oxaliplatin (10 $\mu \mathrm{M})$. B. Cleavage of caspase-9 and caspase-3 in the SW480/OR and HT29/OR cells treated with miR-122 and oxaliplatin (10 $\mu$ M). C. Flow cytometry analysis was performed to detect the apoptotic rates of the SW480/OR and HT29/OR cells treated with miR-122 and oxaliplatin $(10 \mu \mathrm{M})$. ${ }^{*} \mathrm{p}<0.05$ vs. the oxaliplatin $+\mathrm{NCO}$ group. ${ }^{*} \mathrm{p}<0.05$ vs. the oxaliplatin + miR-122 group. 
the tumor that originated from SW480 cells stably expressing miR-122 (Fig. 6A). In addition, consistent with the preceding results, we observed a negative correlation of miR-122 and XIAP in CRC tumors from SW480/OR (Fig. 6B and 6C). We therefore demonstrated that miR122 inhibited the expression of XIAP and enhanced the anti-tumor effects of oxaliplatin in oxaliplatin-resistant CRC in vivo.

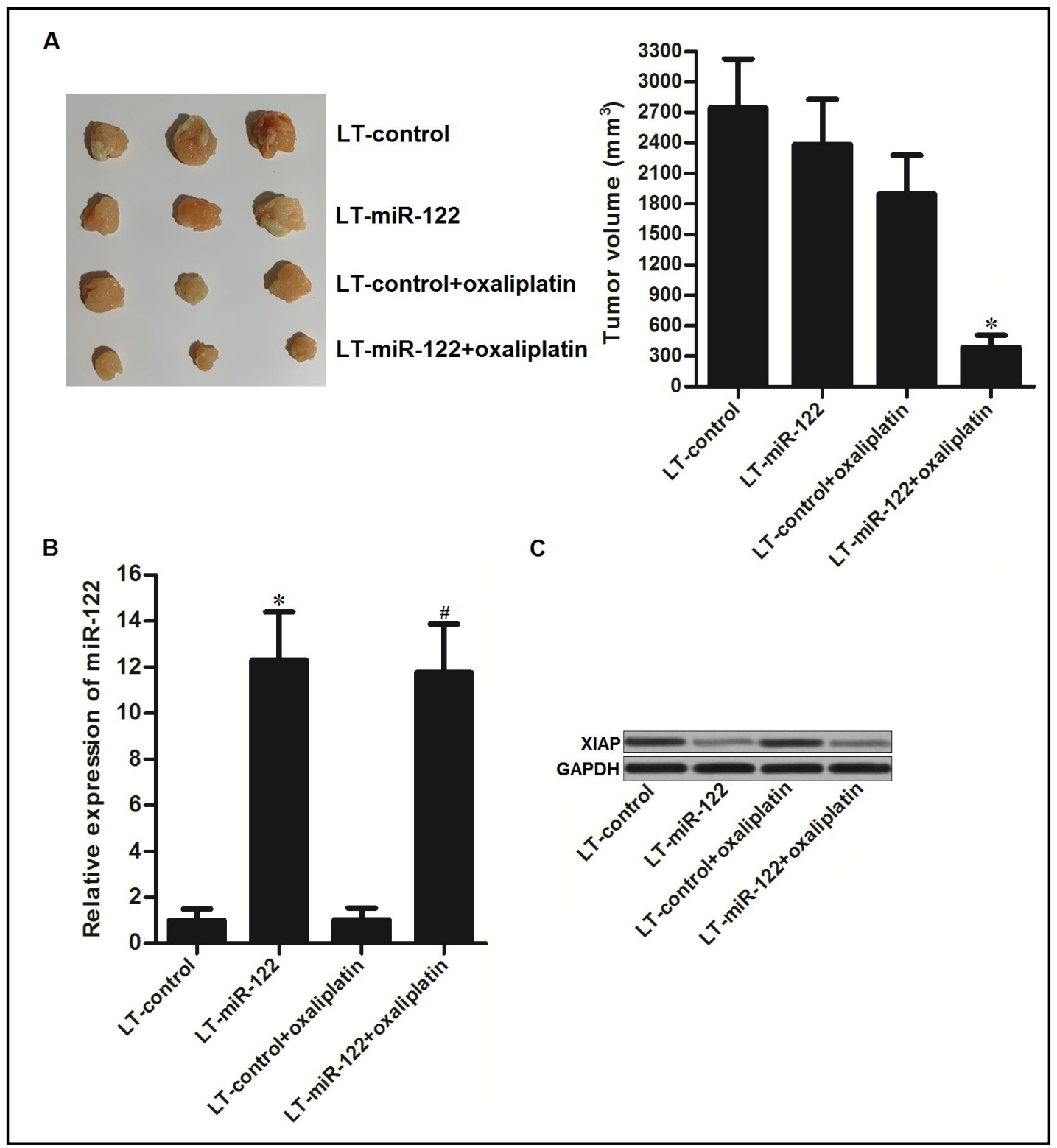

Fig. 6. miR-122 enhanced the anti-tumor effect of oxaliplatin against CRC. A. After inoculation (28 days), mice ( $n=28,8$ mice in each group) treated with oxaliplatin i.p. twice a week ( $5 \mathrm{mg} / \mathrm{kg}$ ) were sacrificed and the tumor tissues were harvested. ${ }^{*} \mathrm{p}<0.05$ vs. the LT-control + oxaliplatin group. B. Expression of miR-122 in separated xenografts detected by qRT-PCR. ${ }^{*} \mathrm{p}<0.05$ vs. the LT-control group. ${ }^{*} \mathrm{p}<0.05$ vs. the LT-control + oxaliplatin group. C. Expression of XIAP in separate xenografts evaluated by western blot analysis. 


\section{Discussion}

Although surgery is the first choice for the treatment of CRC, tumors are unresectable among patients with metastatic disease $[22,23]$. Oxaliplatin-based chemotherapy is the firstline treatment for advanced CRC. However, long-term use usually decreases drug-sensitivity among cancer cells, and the development of acquired drug resistance remains a major obstacle [24-26]. The mechanisms that determine oxaliplatin sensitivity in CRC have not been identified. Many studies have shown that XIAP is usually overexpressed in association with chemoresistance in cancer cells. XIAP is a key member of anti-apoptotic protein, which can prevent the activation of caspases by interacting with them [27-29]. Therefore, the overexpression of XIAP decreases the sensitivity of cancer cells to apoptotic signals. Furthermore, research has revealed that the overexpression of XIAP also can contribute to the resistance of cancer cells during chemotherapy. In clinical terms, a high expression level of XIAP predicts a poor prognosis in many cancers [30-33]. XIAP is considered as an important target for enhancing chemotherapy-induced apoptosis in cancer cells.

In this study, we established oxaliplatin-resistant CRC models in SW480 and HT29 cell lines. Our results showed that the established SW480/OR and HT29/OR cells were resistant to oxaliplatin treatment. Mechanistically, we proved that the overexpression of XIAP was responsible for oxaliplatin resistance in oxaliplatin-resistant CRC models. On the other hand, increased expression of XIAP decreased the sensitivity of oxaliplatin in parental SW480 and HT29 cells. We demonstrated that the overexpression of XIAP contributed to the acquired resistance against oxaliplatin in CRC.

Recent reports have shown that acquired chemoresistance is usually induced by the dysregulation of miRNAs in many cancers. Among these dysregulated miRNAs, miR-122, which has been proven to be a tumor suppressor, is usually downregulated in multiple cancers, including CRC. The absence of miR-122 is found to enhance the proliferation, metastatic, and tumorigenic properties of cancer cells [34,35]. On the other hand, the recovery of miR-122 expression has been reported to increase the chemosensitivity of cancer cells [36, 37]. The overexpression of miR-122 may represent a potential strategy for cancer treatments.

In this study, a significant decrease in miR-122 expression was observed in oxaliplatinresistant CRC cells compared with the parental CRC cells. Furthermore, we proved that the overexpression of XIAP was induced by the downregulation of miR-122 in oxaliplatinresistant CRC cells. Interestingly, we found that transfection with exogenous miR-122 weakened the oxaliplatin resistance in SW480/OR and HT29/OR cells through the suppression of the XIAP/caspases pathway. miR-122 was found to function as a sensitizer to oxaliplatin-induced apoptosis by targeting XIAP.

\section{Conclusion}

We have demonstrated the value of adjuvant therapy with miR-122 in reversing oxalipaltin resistance in CRC. Although further studies are required to test the clinical prospects, a combination study with miR-122 may offer a potential approach toward attenuating the chemoresistance of CRC cells against oxaliplatin.

\section{Acknowledgements}

This study was supported by the National Nature Science Foundation of China (grant nos. 81673749, 81673746, 81603439 and 81873122), the Development Action Plan of T.C.M. of the Shanghai Municipal Commission of Health and Family Planning (grant nos. ZY3CCCX-3-3031 and ZY3-LCPT-2-1001), the Medical Guide Project of the Shanghai Municipal 
Commission for Science and Technology (grant no. 15401932400), and the "Xinglin Star" plan of the Shanghai Municipal Commission of Health and Family Planning (grant no. ZY3RCPY-2-2023).

\section{Disclosure Statement}

The authors have no conflicts of interest.

\section{References}

1 Siegel RL, Miller KD, Jemal A: Cancer statistics, 2015. CA Cancer J Clin 2015;65:5-29.

-2 Manfredi S, Lepage C, Hatem C, Coatmeur O, Faivre J, Bouvier AM: Epidemiology and management of liver metastases from colorectal cancer. Ann Surg 2006;244:254-259.

-3 Kabolizadeh P, Engelmann BJ, Pullen N, Stewart JK, Ryan JJ, Farrell NP: Platinum anticancer agents and antidepressants: desipramine enhances platinum-based cytotoxicity in human colon cancer cells. J Biol Inorg Chem 2012;17:123-132.

4 Zhou J, Li P, Xue X, He S, Kuang Y, Zhao H, Chen S, Zhi Q, Guo X: Salinomycin induces apoptosis in cisplatinresistant colorectal cancer cells by accumulation of reactive oxygen species. Toxicol Lett 2013;222:139145.

5 Zhou Y, Wan G, Spizzo R, Ivan C, Mathur R, Hu X, Ye X, Lu J, Fan F, Xia L, Calin GA, Ellis LM, Lu X: miR-203 induces oxaliplatin resistance in colorectal cancer cells by negatively regulating ATM kinase. Mol Oncol 2014;8:83-92.

6 Bartel DP: MicroRNAs: genomics, biogenesis, mechanism, and function. Cell 2014;116:281-297.

7 Bartel DP: MicroRNAs: target recognition and regulatory functions. Cell 2009;136:215-233.

8 Di Leva G, Garofalo M, Croce CM: MicroRNAs in cancer. Annu Rev Pathol 2014;9:287-314.

$>9$ Tian L, Zhang J, Ren X, Liu X, Gao W, Zhang C, Sun Y, Liu M: Overexpression of miR-26b decreases the cisplatin-resistance in laryngeal cancer by targeting ATF2. Oncotarget 2017;8:79023-79033.

10 Hong L, Yang Z, Ma J, Fan D: Function of miRNA in controlling drug resistance of human cancers. Curr Drug Targets 2013;14:1118-1127.

11 Haenisch S, Cascorbi I: miRNAs as mediators of drug resistance. Epigenomics 2012;4:369-381.

12 Wang D, Lippard SJ: Cellular processing of platinum anticancer drugs. Nat Rev Drug Discov 2005;4:307320 .

13 Mowaka S, Ziehe M, Mohamed D, Hochkirch U, Thomale J, Linscheid MW: Structures of oxaliplatinoligonucleotide adducts from DNA. J Mass Spectrom 2012;47:1282-1293.

14 Ginés A, Bystrup S, Ruiz de Porras V, Guardia C, Musulén E, Martínez-Cardús A, Manzano JL, Layos L, Abad A, Martínez-Balibrea E: PKM2 Subcellular Localization Is Involved in Oxaliplatin Resistance Acquisition in HT29 Human Colorectal Cancer Cell Lines. PLoS One 2015;10:e0123830.

15 Hu CJ, Wang B, Tang B, Chen BJ, Xiao YF, Qin Y, Yong X, Luo G, Zhang JW, Zhang D, Li S, He F, Yang SM: The FOXM1-induced resistance to oxaliplatin is partially mediated by its novel target gene Mcl-1 in gastric cancer cells. Biochim Biophys Acta 2015;1849:290-299.

16 Jin YY, Chen QJ, Xu K, Ren HT, Bao X, Ma YN, Wei Y, Ma HB: Involvement of microRNA-141-3p in 5 -fluorouracil and oxaliplatin chemo-resistance in esophageal cancer cells via regulation of PTEN. Mol Cell Biochem 2016;422:161-170.

17 Li Y, Jian Z, Xia K, Li X, Lv X, Pei H, Chen Z, Li J: XIAP is related to the chemoresistance and inhibited its expression by RNA interference sensitize pancreatic carcinoma cells to chemotherapeutics. Pancreas 2006;32:288-296.

18 Miyamoto M, Takano M, Iwaya K, Shinomiya N, Kato M, Aoyama T, Sasaki N, Goto T, Suzuki A, Hitrata J, Furuya K: X-chromosome-linked inhibitor of apoptosis as a key factor for chemoresistance in clear cell carcinoma of the ovary. Br J Cancer 2014;110:2881-2886.

19 Nestal de Moraes G, Delbue D, Silva KL, Robaina MC, Khongkow P, Gomes AR, Zona S, Crocamo S, Mencalha AL, Magalhães LM, Lam EW, Maia RC: FOXM1 targets XIAP and Survivin to modulate breast cancer survival and chemoresistance. Cell Signal 2015;27:2496-2505. 


\section{Cellular Physiology Cell Physiol Biochem 2018;51:2148-2159 and Biochemistry DOl: 10.1159/000495832 2018 穴 2018 The Author(s). Published by S. Karger AG, Basel

Hua et al.: miR-122 Reduces Oxaliplatin Resistance in CRC

20 Devi GR: XIAP as target for therapeutic apoptosis in prostate cancer. Drug News Perspect 2004;17:127-134.

21 Kashkar H: X-linked inhibitor of apoptosis: a chemoresistance factor or a hollow promise. Clin Cancer Res 2010;16:4496-4502.

22 Brandi G, De Lorenzo S, Nannini M, Curti S, Ottone M, Dall'Olio FG, Barbera MA, Pantaleo MA, Biasco G: Adjuvant chemotherapy for resected colorectal cancer metastases: Literature review and meta-analysis. World J Gastroenterol 2016;22:519-533.

23 Brouquet A, Nordlinger B: Neoadjuvant and adjuvant therapy in relation to surgery for colorectal liver metastases. Scand J Gastroenterol 2012;47:286-295.

24 Petrelli F, Coinu A, Ghilardi M, Cabiddu M, Zaniboni A, Barni S: Efficacy of oxaliplatin-based chemotherapy + bevacizumab as first-line treatment for advanced colorectal cancer: a systematic review and pooled analysis of published trials. Am J Clin Oncol 2015;38:227-233.

25 Watanabe K, Kawahara H, Enomoto H, Toyama Y, Akiba T, Yanaga K: Feasibility study of oxaliplatin with oral S-1 or capecitabine as first-line therapy for patients with metastases from colorectal cancer. Anticancer Res 2013;33:4029-4032.

26 Temraz S, Mukherji D, Alameddine R, Shamseddine A: Methods of overcoming treatment resistance in colorectal cancer. Crit Rev Oncol Hematol 2014;89:217-230.

27 Rodríguez-Berriguete G, Torrealba N, Ortega MA, Martínez-Onsurbe P, Olmedilla G, Paniagua R, Guil-Cid M, Fraile B, Royuela M: Prognostic value of inhibitors of apoptosis proteins (IAPs) and caspases in prostate cancer: caspase- 3 forms and XIAP predict biochemical progression after radical prostatectomy. BMC Cancer 2015;15:809.

28 Checinska A, Hoogeland BS, Rodriguez JA, Giaccone G, Kruyt FA: Role of XIAP in inhibiting cisplatininduced caspase activation in non-small cell lung cancer cells: a small molecule Smac mimic sensitizes for chemotherapy-induced apoptosis by enhancing caspase-3 activation. Exp Cell Res 2007;313:1215-1224.

-29 Shiozaki EN, Chai J, Rigotti DJ, Riedl SJ, Li P, Srinivasula SM, Alnemri ES, Fairman R, Shi Y: Mechanism of XIAP-mediated inhibition of caspase-9. Mol Cell 2003;11:519-527.

30 Li Y, Jian Z, Xia K, Li X, Lv X, Pei H, Chen Z, Li J: XIAP is related to the chemoresistance and inhibited its expression by RNA interference sensitize pancreatic carcinoma cells to chemotherapeutics. Pancreas 2006;32:288-296.

-31 Han J, Liu Z, Wang N, Pan W: MicroRNA-874 inhibits growth, induces apoptosis and reverses chemoresistance in colorectal cancer by targeting X-linked inhibitor of apoptosis protein. Oncol Rep 2016;36:542-550.

-32 Seligson DB, Hongo F, Huerta-Yepez S, Mizutani Y, Miki T, Yu H, Horvath S, Chia D, Goodglick L, Bonavida B: Expression of X-Linked Inhibitor of Apoptosis Protein Is a Strong Predictor of Human Prostate Cancer Recurrence. Clin Cancer Res 2007;13:6056-6063.

-33 Tamm I, Kornblau SM, Segall H, Krajewski S, Welsh K, Kitada S, Scudiero DA, Tudor G, Qui YH, Monks A, Andreeff M, Reed JC: Expression and Prognostic Significance of IAP-Family Genes in Human Cancers and Myeloid Leukemias. Clin Cancer Res 2000;6:1796-1803.

-34 Tsai WC, Hsu PW, Lai TC, Chau GY, Lin CW, Chen CM, Lin CD, Liao YL, Wang JL, Chau YP, Hsu MT, Hsiao M, Huang HD, Tsou AP: MicroRNA-122, a tumor suppressor microRNA that regulates intrahepatic metastasis of hepatocellular carcinoma. Hepatology 2009;49:1571-1582.

-35 Qin H, Sha J, Jiang C, Gao X, Qu L, Yan H, Xu T, Jiang Q, Gao H: miR-122 inhibits metastasis and epithelialmesenchymal transition of non-small-cell lung cancer cells. Onco Targets Ther 2015;8:3175-3184.

-36 Pan C, Wang X, Shi K, Zheng Y, Li J, Chen Y, Jin L, Pan Z: MiR-122 Reverses the Doxorubicin-Resistance in Hepatocellular Carcinoma Cells through Regulating the Tumor Metabolism. PLoS One 2016;11:e0152090.

-37 Xu Y, Huang J, Ma L, Shan J, Shen J, Yang Z, Liu L, Luo Y, Yao C, Qian C: MicroRNA-122 confers sorafenib resistance to hepatocellular carcinoma cells by targeting IGF-1R to regulate RAS/RAF/ERK signaling pathways. Cancer Lett 2016;371:171-181. 